\title{
IB-LBM study on cell sorting by pinched flow fractionation
}

\author{
Jingtao $\mathrm{Ma}^{\mathrm{a}}$, Yuanqing $\mathrm{Xu}^{\mathrm{a}, *}$, Fangbao Tian ${ }^{\mathrm{b}}$ and Xiaoying Tang ${ }^{\mathrm{a}}$ \\ ${ }^{a}$ School of Life Science, Beijing Institute of Technology, Beijing 100081, China \\ ${ }^{\mathrm{b}}$ School of Engineering and Information Technology, University of New South Wales, Canberra, ACT \\ 2600, Australia
}

\begin{abstract}
Separation of two categories of cells in pinched flow fractionation(PFF) device is simulated by employing IB-LBM. The separation performances at low Reynolds number (about 1) under different pinched segment widths, flow ratios, cell features, and distances between neighboring cells are studied and the results are compared with those predicted by the empirical formula. The simulation indicates that the diluent flow rate should approximate to or more than the flow rate of particle solution in order to get a relatively ideal separation performance. The discrepancy of outflow position between numerical simulation and the empirical prediction enlarges, when the cells become more flexible. Too short distance between two neighboring cells could lead to cell banding which would result in incomplete separation, and the relative position of two neighboring cells influences the banding of cells. The present study will probably provide some new applications of PFF, and make some suggestions on the design of PFF devices.
\end{abstract}

Keywords: IB-LBM, pinched flow fractionation, cell separation

\section{Introduction}

It is very significant to separate various categories of particles in the chemical and medical areas, for example, cells and polymer beads. With the development of the microfluidics, it is more and more convenient to perform particles isolation utilizing micro devices [1-4]. Pinched flow fractionation (PFF) introduced by Yamada et al. [5] is a novel technique to separate particles continuously based on particle size. Experiment method has been employed to separate ploy beads, emulsion droplets [6], and erythrocytes [7]. In the study of Takagi et al. [7], the erythrocytes are separated from the blood which demonstrates the effectivity of PFF to sort cells. Moreover, numerical method has been used to study this technique systematically as well. For example, Shardt et al. [8] employed the lattice Boltzmann method to study microsphere motion in the PFF device. However, they studied merely one particle every time which may not be accurate enough to analyze the separation performance of PFF, and the interaction between particles may significantly affect the performance of PFF has not been considered.

\footnotetext{
*Corresponding author: Yuanqing Xu, School of Life Science, Beijing Institute of Technology, Beijing 100081, China. Tel.:010-68912393; E-mail: bitxyq@bit.edu.cn
}

0959-2989/14/\$27.50 @ 2014 - IOS Press and the authors. 
In recent years, the immersed boundary-Lattice Boltzmann method (IB-LBM) have received considerable attention in simulating the movements of complex boundaries. By utilizing the numerical frame of IB-LBM, it is proved that the dynamical behaviors of cells in flow, such as the cell motion, deformation and interaction, can be simulated successfully $[9,14]$. In this paper, the 2D IB-LBM is adopted to model the dynamic behaviors of a certain amount of cells in a PFF device, where the cell diameters are set with two different sizes randomly. Based on this model, the cell sorting principle, which is related to the cell membrane stiffness, the channel sizes and the flow distribution are studied numerically.

\section{Models and methods}

\subsection{Mathematical models}

In the numerical model, the fluid motion is solved by LBM with D2Q9 lattice model.The discrete lattice Boltzmann equation of a single relaxation time model is [9-11]

$$
g_{i}\left(\boldsymbol{x}+\boldsymbol{e}_{i} \Delta t, t+\Delta t\right)-g_{i}(\boldsymbol{x}, t)=-\frac{1}{\tau}\left[g_{i}(\boldsymbol{x}, t)-g_{i}^{e q}(\boldsymbol{x}, t)\right]+\Delta t G_{i}
$$

where $g_{i}(\boldsymbol{x}, t)$ is the distribution function for particles of velocity $\boldsymbol{e}_{i}$ at position $\boldsymbol{x}$ and time $t, \Delta t$ is the time step, $g_{i}^{e q}(\boldsymbol{x}, t)$ is the equilibrium distribution function, $\tau$ is the nondimensional relaxation time, and $G_{i}$ is the body force term. $g_{i}^{e q}$ and $G_{i}$ are calculated by [12]

$$
g_{i}^{e q}=\omega_{i} \rho\left[1+\frac{\boldsymbol{e}_{i} \cdot \boldsymbol{u}}{c_{s}^{2}}+\frac{\boldsymbol{u} \boldsymbol{u}:\left(\boldsymbol{e}_{i} \boldsymbol{e}_{i}-c_{s}^{2} \mathbf{I}\right)}{2 c_{s}^{4}}\right], \quad G_{i}=\left(1-\frac{1}{2 \tau}\right) \omega_{i}\left[\frac{\boldsymbol{e}_{i}-\boldsymbol{u}}{c_{s}^{2}}+\frac{\boldsymbol{e}_{i} \cdot \boldsymbol{u}}{c_{s}^{4}} \boldsymbol{e}_{i}\right] \cdot \boldsymbol{f}
$$

in which $\boldsymbol{u}$ is the velocity vector, $\boldsymbol{f}$ is the body force density vector, $\rho$ is the density, $\mu$ is the viscosity, and $\omega_{i}$ are the weights defined by $\omega_{0}=4 / 9, \omega_{i}=1 / 9$ for $i=1$ to 4 and $\omega_{i}=1 / 36$ for $i=5$ to $8 . C_{s}=\Delta x / \sqrt{3} \Delta t$ is the sound speed.

For the immersed boundary to model the cell membrane, its position can be updated within one time step of $\Delta t$ through [13]

$$
\boldsymbol{U}(s, t)=\int_{\Omega} \boldsymbol{u}(\boldsymbol{x}, t) D(\boldsymbol{x}-\boldsymbol{X}(s, t)) \mathrm{d} \boldsymbol{x}, \text { and } \frac{\partial \boldsymbol{X}}{\partial t}=\boldsymbol{U}(s, t),
$$

where $\boldsymbol{X}(s, t)$ is the position of the membrane $s$ at time $t . \boldsymbol{U}(s, t)$ is the velocity of membrane and $\boldsymbol{u}(\boldsymbol{x}, t)$ is the velocity of fluid. $\mathrm{d} \boldsymbol{x}$ is the lattice side length, $\Omega$ is the nearby area of the membrane controlled by a Delta function $D(\boldsymbol{x}-\boldsymbol{X})[13,14]$. In present studies, the cell membrane mechanics is proposed as $\boldsymbol{F}=\boldsymbol{F}_{s}-\boldsymbol{F}_{b}+\boldsymbol{F}_{a}+\boldsymbol{F}_{e}$, in which $\boldsymbol{F}_{s}$ is the tensile force, $\boldsymbol{F}_{b}$ the bending force, $\boldsymbol{F}_{a}$ the normal force on the membrane which controls the cell incompressibility and $\boldsymbol{F}_{e}$ is the cell-wall extrusion acting on the membrane. The four force components are $[9,11,15,16]$ 


$$
\begin{aligned}
& \boldsymbol{F}_{s}=\frac{\partial}{\partial s}\left[K_{s}\left(\left|\frac{\partial \boldsymbol{X}(s, t)}{\partial s}\right|-1\right) \frac{\partial \boldsymbol{X}(s, t)}{\partial s}\right], \quad \boldsymbol{F}_{b}=K_{b} \frac{\partial^{4} \boldsymbol{X}(s, t)}{\partial s^{4}}, \quad \boldsymbol{F}_{a}=K_{a} \frac{S-S_{0}}{S_{0}} \mathbf{n} \quad \text { and } \\
& \boldsymbol{F}_{e}= \begin{cases}K_{e} \frac{\boldsymbol{X}(s, t)-\boldsymbol{X}_{w}}{\left(\min \left(\left|\boldsymbol{X}(s, t)-\boldsymbol{X}_{w}\right|\right)\right)^{3}}, & \left|\boldsymbol{X}(s, t)-\boldsymbol{X}_{w}\right| \leq r_{c}, \\
0, & \left|\boldsymbol{X}(s, t)-\boldsymbol{X}_{w}\right|>r_{c} .\end{cases}
\end{aligned}
$$

In Eq. (4), $S$ in the formula of $\boldsymbol{F}_{s}$ is the cell area and $S_{0}$ is the reference to RBC area. In the formula of $\boldsymbol{F}_{e}$, $\boldsymbol{X}_{w}$ is the position of the vessel wall, and $r_{c}$ is the cut-off distance of the effective scope in the membranewall interaction. $K_{l}, K_{b}, K_{s}$ and $K_{e}$ are the constant coefficients for the corresponding force components. The dimensionless bending rigidity $K_{b}$ ranges from 0.05 to $1.8[15,19]$. The dimensionless extensional rigidity $K_{l}$ is set to the same to $K_{b}$ which can ensure the normal deformation of cells. Moreover, $K_{s}, K_{e}$ is $5 \times 10^{-3}$ and $1 \times 10^{-8}$, respectively. Then the force density $\boldsymbol{f}$ in Eq. (2) acting on the nearby fluid is calculated by $[13,15-18]$

$$
\boldsymbol{f}(\boldsymbol{x}, t)=\int_{\Gamma} \boldsymbol{F}(s, t) D(\boldsymbol{x}-\boldsymbol{X}(s, t)) d s
$$

\subsection{Physical model and simulation setup}

The primary schematic structure of the PFF device is illustrated in the Figure 1. There is no solution in the device initially, then solution is released in it to form a stable flow field. Two categories of cells are released into inlet 1 one by one, flow with the solution, separate at the expanded segment and finally get out of the device. The outflow positions of the center of cells are recorded, averaged by category and represented by $y_{p}$. Velocity inlet boundary conditions are used to define the fluid velocity at flow inlets, pressure outlet boundary conditions are employed to define the outlets, and the upper and lower boundary is set to be walls. The Reynolds number of the flow is about 1 in this simulation. The flow of inlet 1 is about $0.53 \mathrm{~m}^{3} / \mathrm{s}$, and the fundamental flow ratio of two inlets $\left(Q_{1}: Q_{2}\right)$ is 1:6.

The LBM and actual size of the main parameters are listed as the following. Actual size: $x_{0}=640$ $\mu m, y_{0}=320 \mu m, x_{p}=50 \mu m, w_{0}=20 \mu m, x_{e}=440 \mu m$. LBM size: $x_{0}=800, y_{0}=400, x_{p}=62$, $w_{0}=24, x_{e}=550$.

Table 1

Validation with previous studies

\begin{tabular}{lllllllll}
\hline \multicolumn{2}{l}{ pinched segment width $w_{p}(\mu m)$} & \multicolumn{2}{l}{ outflow position $y_{p} / y_{0}$ (Small) } & \multicolumn{2}{c}{ outflow position $y_{p} / y_{0}$ (Large) } \\
Ref [8] & Ref [5] & present & Ref [8] & Ref [5] & present & Ref [8] & Ref [5] & present \\
\hline 45 & 47 & 48 & 0.868 & $0.87 \pm 0.01$ & 0.838 & 0.711 & $0.66 \pm 0.01$ & 0.732 \\
55 & 56 & 56 & 0.891 & $0.89 \pm 0.02$ & 0.837 & 0.769 & $0.76 \pm 0.02$ & 0.782 \\
85 & 82 & 82 & 0.897 & $0.89 \pm 0.03$ & 0.836 & 0.850 & $0.81 \pm 0.02$ & 0.827 \\
\hline
\end{tabular}




\section{Results and discussion}

\subsection{Validation}

The validation is mainly performed between present simulation results and existing results which encompass the previous experimental and simulation results. The present channel structure and dimension in this section are set approximately the same as those utilised in the Shardt's study [8]. Compared to their study, rigid spherical particles are supplanted by the elastic cells, and the pinched segment widths were set to 48,56 , and $82 \mu \mathrm{m}$, compared to 45,56 and $85 \mu \mathrm{m}$ in their study. Table 1 exhibits the comparison of the simulation results with results of Shardt et al. [8] and the experimental results of Yamada et al. [5].

As displayed in Table 1, the present results agree with the previous works, with little difference from the previous results. The divergence may result from the fact that this paper simulates the movement of cells rather than the rigid spheres whose mechanic property may not be same to the cells.

\subsection{Different pinched segment widths}

In this section, the separation performances of different $w_{0}$ are studied. In the present study, the pinched segment width $w_{0}$ ranges from $20 \mu \mathrm{m}$ to $32 \mu \mathrm{m}$ with an increment of $1.5 \mu \mathrm{m}$. The flow ratio of inlet 1 to inlet $2\left(Q_{1}: Q_{2}\right)$ is 1:6 here which is enough to get a manifest separation effect(Section 3.3). The bending rigidity $K_{b}$ and the extensional rigidity $K_{l}$ are set to the same value of 1.5 , which is to ensure the cells are not too flexible and the structure is stable enough. Yamada et al. [5] proposed a empirical formula to predict to the outflow position of particles in PFF, which is

$$
y_{p}=\frac{y_{0}}{w_{0}}\left(w_{0}-\frac{D}{2}\right)
$$

where $w_{0}$ and $y_{0}$ are the widths of the pinched and expanded segment, $y_{p}$ is the outflow position of particles, and $D$ is the particle diameter.

Figure 2 exhibits the separation performances for certain $w_{p}(20,32 \mu m$, individually), and Figure 3 shows the contrast between the simulation results and the calculated results utilizing Eq. (6). As can be obtained from the figures, it is evident that a better separation consequence can be accomplished,

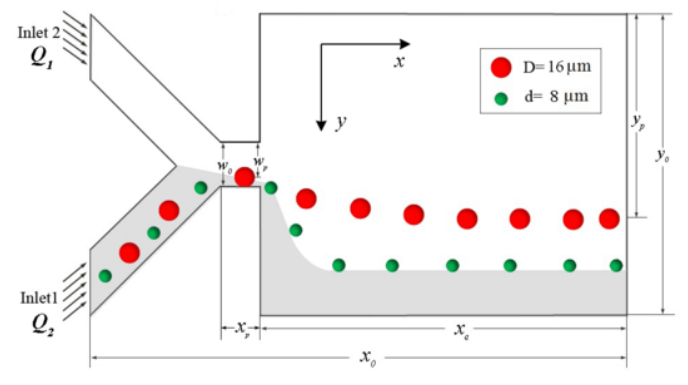

Fig. 1. The basic schematic structure of the simulated device.

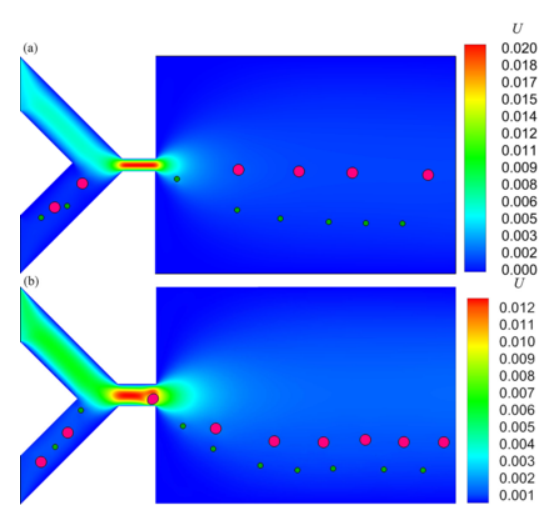

Fig. 2. The separation performances for some $w_{p}$ : (a) $20 \mu m$,(b) $32 \mu m$. 


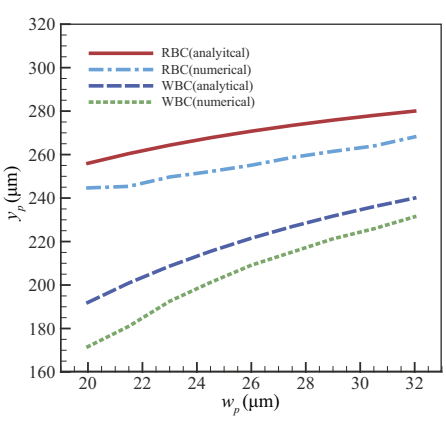

Fig. 3. The outflow position for each $w_{p}$.

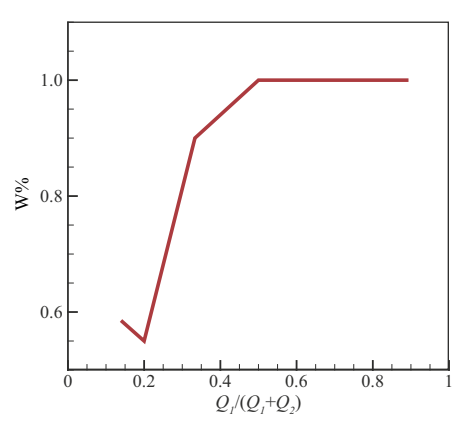

Fig. 4. Percent of complete separation of different flow ratios.

when the pinched segment $w_{p}$ becomes narrower. Furthermore, the calculated values exceed the simulated ones, which is similar to what Vig and Kristensen observed and discussed [20]. The maximum discrepancy between the simulation results and the calculated ones is about $8 \%$.

\subsection{Different flow ratio}

In this section, the separation performances on different flow ratios of inlet 1 to inlet 2 are discussed. In order to get a distinct exhibition, the pinched segment width is set to $20 \mu \mathrm{m}$ (section 3.2). The flow ratio $Q_{1}: Q_{2}=6: 1,4: 1,2: 1,1: 1,1: 2,1: 4,1: 6$, and 1:8 separately. Still the bending rigidity $K_{b}$ and the extensional rigidity $K_{l}$ are set to 1.5 . Here the percent of complete separation is defined as

$$
W \%=\frac{N_{\text {sep }}}{N_{\text {total }}} \times 100 \%,
$$

where $N_{\text {sep }}$ is the number of small cells whose outflow position $y_{p}$ is larger than the average position of large cells, $N_{\text {total }}$ is the total number of small cells.

Figure 4 displays the percent of complete separation. Figure 5(a) shows the outflow positions of two categories of cells in each flow ratio. The difference of outflow position between the small cells and the large ones is exhibited in Figure 5(b), and represented by $\Delta y_{p}$.

As exhibited in Figure 4, two categories of cells can not be separated completely when $Q_{2}$ is less than $Q_{1}$. Then in order to obtain an ideal separation result, $Q_{2}$ should surpass $Q_{1}$. As shown in Figure 5, the outflow position of small cells varies very little, when $Q_{2}$ is amplified to $4 Q_{1}$. It means that it is no more effective to get a better separation by enhancing the flow ratio when $Q_{2}$ augments to some specific value.

\subsection{Different cell membrane stiffness}

In this section, the effect of different cell membrane stiffness on the separation of two categories of cells is studied, where the membrane stiffness includes the elasticity and flexibility. in the present setting, the pinched segment width is still set to $20 \mu \mathrm{m}$, and the flow ratio of the inlet 1 to inlet 2 is 1:6. The extensional rigidity $K_{l}$ and the bending rigidity $K_{b}$, correspondingly control the membrane elasticity and flexibility, are regulated to change the stiffness of cells. according to our numerical tests, $K_{l}$ and $K_{b}$ can 
be set to the same values as $0.05,0.1,0.25,0.5,1.0,1.5$, and 1.8 to express the increments of stiffness of the cell membrane. The average outflow positions in each case are shown in Figure 6.

As exhibited in Figure 6, the average outflow position of small cells deviates from the calculated value gradually, when $K_{b}$ and $K_{l}$ decrease. It may be caused by the deformation of cells in the pinched segment. In addition, it is easy to get that the empirical formula is not applicable to predicting the outflow position of cells any more, when the $K_{b}$ and $K_{l}$ are too small.

\subsection{Cells banding}

In the simulation, a phenomenon of cells banding is observed, and it would affect the separation. In order to figure out what contributes to the banding, the positions of small cell and large cell are set as the following. A large cell is put at the center of the channel, and a small cell surrounds the large one with four different positions: back, left, front, right. The distance between two cells $L$ is set to $0.16,0.4,0.64$, $0.88,1.12,1.36,1.6,1.84,2.08$ and $2.32 \mu \mathrm{m}$. The difference of outflow position of two cells for each $L$ is calculated to estimate the banding. The results are shown in Figure 7.

As illustrated in Figure 7, the phenomenon of banding do occurs when $L$ is too small. Moreover, when $L$ enlarges to $1.6 \mu \mathrm{m}$, the difference of outflow position reaches a steady state, and the relative position of two neighboring cells would affect the separation as well. However, it seems that the small cell at the
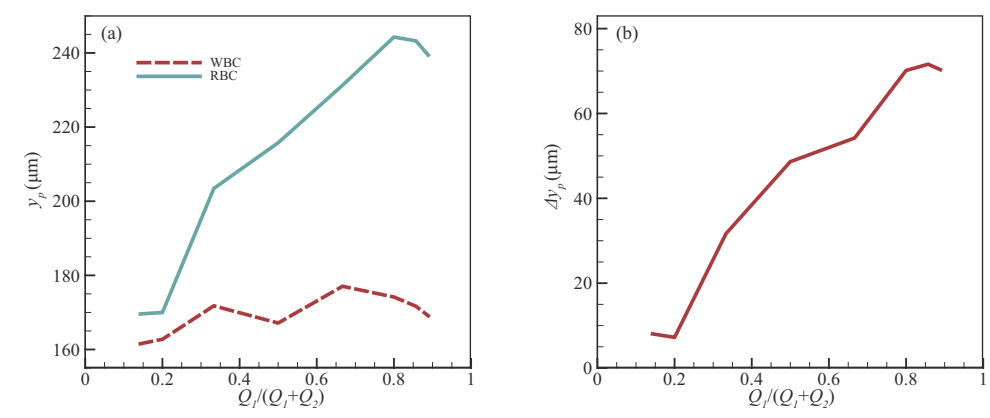

Fig. 5. The average outflow position of different $Q_{2} /\left(Q_{1}+Q_{2}\right)$.

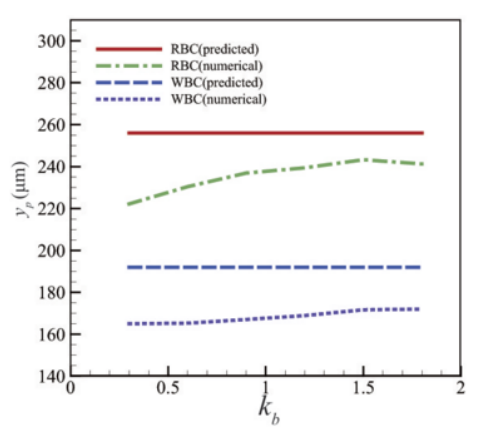

Fig. 6. The outflow position of different cell features. Fig. 7. The differences of outflow positions of different $L$ for each

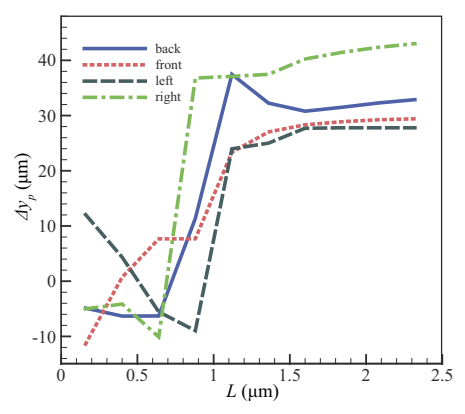
position. 
back, front, and left position reach the approximate separation performance finally, and the right position get a better separation performance.

\section{Conclusion}

The 2D simulation of large numbers of two categories of cells in the PFF device is conducted by utilizing IB-LBM. The study indicates that the flow rate of the dilute solution $\left(Q_{2}\right)$ should be higher than that of the solution containing cells $\left(Q_{1}\right)$, and it is not very effective to get a better separation performance by adding the dilute flow rate when $Q_{2}$ amplifies to $4 Q_{1}$. Our study also reveals that the empirical formula is not applicable to predicting the outflow position any more when the cells is too flexible. Moreover, When two neighboring cells are too close, the phenomenon of cells banding would occur which could result in incomplete separation. The relative position of two cells can influence the banding of cells as well.

\section{Acknowledgement}

This work is supported by the Beijing Higher Education Young Elite Teacher Project(No. YETP1208) and the National Natural Science Foundation of China(No.81301291).

\section{References}

[1] D. Yagmur,O. Ebru,K. Haluk, Dielectrophoresis: Applications and future outlook in point of care, Electrophoresis 34 (2013), 1008-1027.

[2] H. B. Li,R. Bashir, Dielectrophoretic separation and manipulation of live and heat-treated cells of Listeria on microfabricated devices with interdigitated electrodes, Sens. Actuator B-Chem. 86 (2002), 215-221.

[3] M. T. Blom, E. Chmela, R. E. Oosterbroek,R. Tijssen,A. v. d. Berg, On-Chip Hydrodynamic Chromatography Separation and Detection of Nanoparticles and Biomolecules, Anal. Chem. 75 (2003), 6761-6768.

[4] G. Yohannes, M. Jussila, K. Hartonen, M. L. Riekkola, Asymmetrical flow field-flow fractionation technique for separation and characterization of biopolymers and bioparticles, J. Chromatogr. A 1218 (2011), 4104-4116.

[5] M. Yamada, M. Nakashima, M. Seki, Pinched flow fractionation: Continuous size separation of particles utilizing a laminar flow profile in a pinched microchannel, Anal. Chem. 76 (2004), 5465-5471.

[6] H. Maenaka, M. Yamada, M. Yasuda, M. Seki, Continuous and size-dependent sorting of emulsion droplets using hydrodynamics in pinched microchannels, Langmuir 24 (2008), 4405-4410.

[7] J. Takagi, M. Yamada, M. Yasuda, M. Seki, Continuous particle separation in a microchannel having asymmetrically arranged multiple branches, Lab Chip 5 (2005), 778-784.

[8] O. Shardt, S. K. Mitra, J. J. Derksen, Lattice Boltzmann simulations of pinched flow fractionation, Chem. Eng. Sci. 75 (2012), 106-119.

[9] Y. Q. Xu, F. B. Tian, Y. L. Deng, An efficient red blood cell model in the frame of IB-LBM and its application, Int. J. Biomath. 6 (2013), 1250061.

[10] Y. H. Qian, D. Humieres, P. Lallemand, Lattice bgk models for navier-stokes equation, Europhys. Lett. 17 (1992), 479 484.

[11] F. B. Tian, H. Luo, L. Zhu, J. C. Liao, X. Y. Lu, An immersed boundary-lattice Boltzmann method for elastic boundaries with mass, J. Comput. Phys. 230 (2011), 7266-7283.

[12] Z. L. Guo, C. G. Zheng, B. C. Shi, Discrete lattice effects on the forcing term in the lattice boltzmann method, Phys. Rev. E 65 (2002), 046308.

[13] C. S. Peskin, The immersed boundary method, Acta. numerica. 11 (2002), 479-517.

[14] Y. Q. Xu, X. Y. Tang, F. B. Tian, Y. H. Peng, Y. Xu, Y. J. Zeng, IB-LBM simulation of the haemocyte dynamics in a stenotic capillary, Comput. Method Biomec. 17 (2014), 978-985.

[15] F. B. Tian, H. Luo, L. Zhu, X. Y. Lu, Interaction between a flexible filament and a downstream rigid body, Phys. Rev. E 82 (2010), 026301. 
[16] F. B. Tian, Role of mass on the stability of flag/flags in uniform flow, Appl. Phys. Lett. 103 (2013), 034101.

[17] Y. Q. Xu, F. B. Tian, H. J. Li, Y. L. Deng, Red blood cell partitioning and blood flux redistribution in microvascular bifurcation, Theor. Appl. Mech. Lett. 2 (2012), 024001.

[18] F. B. Tian, X. Y. Lu, H. Luo, Propulsive performance of a body with a traveling wave surface, Phys. Rev. E 86 (2012), 016304

[19] L. D. Zhu, G. W. He, S. Z. He, L. Miller, X. Zhang, Q. You, S. F. Fang, An immersed boundary method by the lattice Boltzmann approach in three dimensions with application, Comput. Math. Appl. 61 (2011), 3506-3518.

[20] A. L. Vig, A. Kristensen, Separation enhancement in pinched flow fractionation, Appl. Phys. Lett. 93 (2008), 203507. 